\title{
The Great Breast Cancer Screening Conundrum
}

\author{
Aakash Desai $^{1}$ Aju Mathew 2,3 \\ ${ }^{1}$ Division of Medical Oncology, Department of Internal Medicine, \\ Mayo Clinic, Rochester, Minnesota, United States \\ ${ }^{2}$ Department of Oncology, MOSC Medical College, Kolenchery, \\ Kerala, India \\ ${ }^{3}$ Department of Internal Medicine, Markey Cancer Center, \\ University of Kentucky, Lexington, Kentucky, United States
}

Ind J Med Paediatr Oncol 2021;42:376-379.

Breast cancer screening is a vital health care program delivered at a primary care level. Mammography is often considered synonymous with breast cancer screening. In this viewpoint, we will discuss the fundamental principles of a screening program, the evidence for screening mammography in preventive health care, and critically appraise the utility of a breast cancer screening program in a low-middle income country (LMIC) such as India.

\section{Key Principles of a Screening Program}

The concept of screening is based on the tenet of maximizing benefit and minimizing harm. The obvious benefit of screening is early diagnosis while the harm is overdiagnosis. Overdiagnosis is defined as detecting tumors that might never have progressed to become symptomatic or life-threatening in the absence of screening. A false-positive cancer screening test implies that the test suggested a cancer diagnosis, but in reality, the patient does not have a biopsy-proven malignancy. A false-negative cancer screening test means that the test failed to pick up a malignancy. A good cancer screening test should have both low false-positive and false-negative rates. The former avoids unnecessary procedures and anxieties, while the latter avoids the risk of falsely reassuring a person regarding their health. An ideal cancer screening test should help diagnose aggressive cancers at an earlier stage when it is curable. If such a test is appropriately used in a primary care setting, it should improve survival rates.

\section{Screening Mammography: Is It Beneficial?}

Despite the risk of overdiagnosis, breast cancer screening is widespread in high-income countries like the US due to the enthusiasm about cancer screening and the medicolegal environment, which rewards clinicians' vigilance. ${ }^{1}$ False-positive
Address for correspondence Aju Mathew, MOSC Medical College, Kolenchery, Kerala, India (e-mail: cancerkerala@gmail.com).

mammogram rates in Europe for women undergoing biennial screening from age 50 to 69 years in Europe was found to be approximately $20 \% .{ }^{2}$ False-positive mammogram results in biopsy in 3 to $7 \%{ }^{3}$ In the US, the 10 -year false positive rate was found to be $30 \%{ }^{4}$

False-negative mammograms run the risk of missing a cancer diagnosis when one exists. It is likely to be a problem in younger women with dense breasts. Interval cancers are those cancers detected between two screening mammograms, either due to being overlooked at the last mammogram or due to rapidly growing cancers. Among the breast cancers detected by screening, 10 to $30 \%$ are interval cancers (numbers vary by population). ${ }^{5}$ Loberg et al suggested that for 1,000 women who are 50 years old and above screened every 2 years for 20 years, 200 are false positive, 30 will undergo biopsy due to a false-positive result, 3 will develop an interval cancer, 15 will be overdiagnosed, and only 2 to 3 will be saved from dying of breast cancer. ${ }^{6}$

This begs the following question: Is screening mammography as beneficial as we think it to be?

First, the reduction in breast cancer mortality could be attributable to better systemic therapy than diagnosing the disease at an earlier time point. Older screening trials, which have shown benefit, were from an era of no systemic treatment. Would the use of effective adjuvant treatment erase the presumed benefit of early detection of smaller cancers? A modeling study conducted by Berry et al used seven statistical models to demonstrate that both screening mammography and treatment have helped reduce the death rate from breast cancer in the US. ${ }^{7}$ Furthermore, simulation modeling techniques have estimated that advances in treatment were associated with more significant decreases in breast cancer mortality rates than advances in screening. However, these associations varied by the breast cancer molecular subtype (screening may have a more substantial impact in the
DOI https://doi.org/ $10.1055 / \mathrm{s}-0041-1731859$ ISSN 0971-5851
(C) 2021. Indian Society of Medical and Paediatric Oncology.

This is an open access article published by Thieme under the terms of the Creative Commons Attribution-NonDerivative-NonCommercial-License, permitting copying and reproduction so long as the original work is given appropriate credit. Contents may not be used for commercial purposes, or adapted, remixed, transformed or built upon. (https://creativecommons.org/licenses/by-nc-nd/4.0/).

Thieme Medical and Scientific Publishers Private Ltd. A-12, Second Floor, Sector -2, NOIDA -201301, India 
setting of triple-negative breast cancer). ${ }^{8}$ Nevertheless, these studies do not entirely negate the role of breast cancer screening mammography, although much of the mortality reduction seems to come from adjuvant therapy.

Second, there is no reduction in late-stage breast cancer incidence despite 20 years of screening. ${ }^{9}$ If screening is so effective, why is there no reduction in the incidence of advanced breast cancer? Bleyer et al studied the US Surveillance, Epidemiology, and End Results (SEER) database from 1975 to 2008 and found that patients with late-stage cancer decreased by $8 \%$, from 102 to 94 cases per 100,000 . Hence, only 8 of the 122 early-stage cancers diagnosed with mammography progressed to advanced disease. ${ }^{10}$ This not only reflects the lack of reduction in the incidence of advanced breast cancers despite screening but may also explain the lack of mortality benefit with screening measures.

Third, screening mammography can result in finding cancers that do not affect the disease's natural history (overdiagnosis). Patients may die with such cancers and not of it. It is estimated that around 10 to $40 \%$ of all breast cancers diagnosed in screening mammography trials could be attributed to overdiagnosis. ${ }^{11}$ In 2008 , breast cancer was possibly overdiagnosed in more than 70,000 women, who accounted for $31 \%$ of all breast cancers diagnosed. Between 1979 to 2008, over 1 million cases may have been overdiagnosed, as shown by various estimates. ${ }^{10}$

Finally, the often understated harm of overdiagnosis is the impact of cancer therapy. Cardiac exposure to ionizing radiation can cause a subsequent increase in the risk of ischemic heart disease. ${ }^{12,13}$ Darby et al observed a linear increase in rates of major coronary events with the mean dose by $7.4 \%$ per gray, without any apparent threshold effect. The cardiotoxicity started within the first 5 years after radiotherapy and continued into the third-decade postradiotherapy. ${ }^{14}$ Longer duration of aromatase inhibitor use was also associated with increased odds of developing cardiovascular disease $(\mathrm{OR}=1.26, p<0.001$; number needed to harm-132) and hypercholesterolemia. ${ }^{15}$ These downstream effects of treatment for overdiagnosed cancers can have detrimental effects, often causing more harm than good.

\section{Screening Mammography: Is It Oversold?}

Even if screening is marginally beneficial, are we overselling screening to our patients? A meta-analysis of nine screening mammography trials demonstrated that among women aged 39 to 75 years invited to screen, the prevented fraction of breast cancer mortality at 13-year follow-up was 0.80 (95\% CI, 0.73-0.89), with a relative risk reduction of $20 \%{ }^{3}$ A study was conducted to understand women's perceptions of the effects of mammography screening on breast cancer mortality in the US. Among the 4140 women who participated, $68 \%$ women believed that screening prevents or reduces the risk of contracting breast cancer, $62 \%$ thought that screening at least halves breast cancer mortality, and $75 \%$ believed that 10 years of regular screening would prevent 10 or more breast cancer deaths per 1,000 women. This study demonstrates that most women grossly overestimate the benefits expected from screening mammography compared with its actual effect. ${ }^{16}$ Hence, appropriate communication of likely benefit and harm is required between the physician and patient. ${ }^{6}$

\section{Is Mammography the Be-All and End-All of Screening for Breast Cancer?}

An alternative to screening mammography was suggested by a Canadian trial, which showed that annual mammography in women aged between 40 to 59 years was no better than a physical examination or usual care in terms of mortality reduction, especially in the era of adjuvant therapy. ${ }^{17}$ During the 5-year screening period, 666 invasive breast cancers (among 44,925 participants) were diagnosed in the mammography arm versus 524 (among 44,910 participants) in the control arm. Of these, 180 and 171 women died of breast cancer during the follow-up period in the mammography and the control arms, respectively, with an overall hazard ratio (HR) for death being 1.05 (95\% CI, 0.85-1.30). Thus, the cumulative breast cancer mortality was similar between the two arms (HR: 0.99, [0.88 to 1.12]). After 15 years of follow-up, an excess of 106 cancers was observed in the mammography arm, which can be attributed to overdiagnosis. Thus, this trial provided an alternative solution to screening using mammography. However, it was not incorporated in the most commonly used U.S. Preventive Services Task Force (USPSTF) guidelines, which reports insufficient evidence for or against clinical breast examination (CBE).

The often advertised tagline of "Screening Saves Lives" is likely to be accurate, albeit the quantum of benefit is expected to be minuscule. Screening mammography likely saves lives even with better systemic therapy, but not as much as indicated by the screening trials. One can argue both ways for whether it is reasonable to skip breast cancer screening given its harms. ${ }^{18} \mathrm{~A}$ good annual CBE likely saves lives too. Hence, in our opinion, giving women a choice and engaging in shared decision-making must be unequivocally adopted as the best practice.

\section{Breast Cancer Screening in India}

In 2016, the government of India declared population-wide screening of oral, cervical, and breast cancer for women over 30 years of age..$^{19}$ Breast cancer screening was recommended using $\mathrm{CBE}$ done once in 5 years for women between 30 and 65 years. Such a decision was made primarily to improve India's dismal breast cancer survival outcomes (5-year survival rate is around $65 \%$ compared with around $85 \%$ in the US). Should the government of India have adopted screening mammography instead of CBE? We believe that there is no role for population-wide screening mammography program in India (and likely in other low-middle income countries [LMIC]) for the following reasons.

First, the burden of breast cancer in India and other LMIC is much different from developed countries. There are approximately 150,000 breast cancer cases diagnosed every year in India. ${ }^{20}$ The breast cancer incidence in India is around 
one-fifth of that seen in Western countries. Currently, the burden of breast cancer in India is much less than in countries where screening trials have been conducted.

Second, breast cancer is diagnosed at a much younger age in India than the West (median age of around 50 years). ${ }^{21}$ Younger women will have denser breasts, and in such women, there is a high risk of a false-negative mammogram.

Third, the prevalence of triple-negative breast cancer (TNBC) in India is considerably higher than that seen in Western populations. ${ }^{22}$ As many as one in three women with breast cancer could have a triple-negative disease. This finding has significant clinical relevance, as it may contribute to poor outcomes in patients with breast cancer in India.

Of note, a TNBC diagnosis is also associated with a higher chance for interval cancers missed despite screening efforts. This is attributable to length bias, since triple-negative tumors grow rapidly with little time for preclinical detection. Interval breast cancers have been associated with triple-negative disease $(\mathrm{OR}=2.0)$ and dense breasts. ${ }^{18,23}$

Finally, mammography requires extensive infrastructure, adequate histopathologic services, and appropriate and easily accessible imaging services. Furthermore, there are multiple challenges related to its affordability and scalability.

\section{Clinical Breast Examination May Trump Mammography}

Based on the World Health Organization (WHO), a good screening program should meet 10 principles. $^{24}$ When applied to an Indian setting, breast cancer screening via mammography does not fulfill at least three of these principles: adequate facilities for diagnosis and treatment should be available (a challenge in the current Indian setting due to scarcity of resources described above); the natural history of the disease should be adequately understood (much research is needed to understand the epidemiology of breast cancer in the Indian population); the cost of case findings (including diagnosis and treatment) should be economically balanced with possible expenditure on medical care as a whole (given lack of universal health care and out-of-pocket form of payment, an economic imbalance currently exists).

Therefore, CBE might be the best screening test for the Indian population. Although $\mathrm{CBE}$ has not been shown to reduce mortality, sufficient evidence exists that it shifts the stage distribution of tumors detected toward a lower stage. A cluster randomized controlled trial from Kerala state in India evaluated whether three rounds of triennial CBE can reduce the advanced disease incidence rate. After completing the first round of screening, the age-standardized incidence rates for early-stage (stage IIA or lower) breast cancer were 18.8 and 8.1 per 100,000 women. For advanced-stage (stage IIB or higher) breast cancer, the rates were 19.6 and 21.7 per 100,000 women in the intervention and control arms. ${ }^{25}$ More women in the CBE cohort were diagnosed with breast cancer and at a lower stage. It is difficult to conclude whether the apparent downstaging of the disease will hold up in later rounds of screening as well.

\section{Conclusion}

Currently, population-wide screening mammography has no role in India. The prospect of screening with CBE once in 5 years (as per the current Government policy) is likely to be of little benefit with regard to improvement in survival outcomes. Nevertheless, we cannot negate the fact that such an organized program can raise awareness and improve infrastructure. Thus, this may serve as a silver lining of the government policy. The more significant challenge will be building a health system that will provide high-quality care in an accessible and equitable way. We believe that increasing awareness and regular self-breast examination will promptly provide the greatest bang for the buck, with a clearly defined path to access care.

\section{Conflict of Interest}

None declared.

\section{References}

1 Sawaya GF. Cervical-cancer screening-new guidelines and the balance between benefits and harms. $\mathrm{N}$ Engl J Med 2009;361(26):2503-2505

2 Hofvind S, Organised Mammographic Screening-More Benefits Than Harms Tidsskrift Den Norske Legeforening; 2013 Mar 19;133(6):619-20. English, Norwegian. doi: 10.4045/ tidsskr.12.0911. PMID: 23552154.

3 Independent UK Panel on Breast Cancer Screening. The benefits and harms of breast cancer screening: an independent review. Lancet 2012;380(9855):1778-1786

4 Oeffinger KC, Fontham ET, Etzioni R, et al. American Cancer Society. Breast cancer screening for women at average risk: 2015 guideline update from the American Cancer Society. JAMA 2015;314(15):1599-1614

5 Törnberg S, Kemetli L, Ascunce N, et al. A pooled analysis of interval cancer rates in six European countries. Eur J Cancer Prev 2010;19(2):87-93

6 Løberg M, Lousdal ML, Bretthauer M, Kalager M. Benefits and harms of mammography screening. Breast Cancer Res 2015;17(1):63

7 Berry DA, Cronin KA, Plevritis SK, et al. Cancer Intervention and Surveillance Modeling Network (CISNET) Collaborators. Effect of screening and adjuvant therapy on mortality from breast cancer. N Engl J Med 2005;353(17):1784-1792

8 Plevritis SK, Munoz D, Kurian AW, et al. Association of screening and treatment with breast cancer mortality by molecular subtype in US women, 2000-2012. JAMA 2018;319(2):154-164

9 Welch HG, Gorski DH, Albertsen PC. Trends in metastatic breast and prostate cancer-lessons in cancer dynamics. N Engl J Med 2015;373(18):1685-1687

10 Bleyer A, Welch HG. Effect of three decades of screening mammography on breast-cancer incidence. $\mathrm{N}$ Engl J Med 2012;367(21):1998-2005

11 Wyld L, Ingram CE. Screening of the population for breast cancer. Surgery 2007;25(6):254-256

12 Lakoski SG, Eves ND, Douglas PS, Jones LW. Exercise rehabilitation in patients with cancer. Nat Rev Clin Oncol 2012;9(5):288-296

13 Patnaik JL, Byers T, DiGuiseppi C, Dabelea D, Denberg TD. Cardiovascular disease competes with breast cancer as the leading cause of death for older females diagnosed with breast cancer: a retrospective cohort study. Breast Cancer Res 2011;13(3):R64 
14 Darby SC, Ewertz M, McGale P, et al. Risk of ischemic heart disease in women after radiotherapy for breast cancer. $\mathrm{N}$ Engl J Med 2013;368(11):987-998

15 Amir E, Seruga B, Niraula S, Carlsson L, Ocaña A. Toxicity of adjuvant endocrine therapy in postmenopausal breast cancer patients: a systematic review and meta-analysis. J Natl Cancer Inst 2011;103(17):1299-1309

16 Bulliard J-L, La Vecchia C, Levi F. Abolishing mammography screening programs? Eur J Cancer Prev 2015;24(4):334

17 Miller AB, Wall C, Baines CJ, Sun P, To T, Narod SA. Twenty five year follow-up for breast cancer incidence and mortality of the Canadian National Breast Screening Study: randomised screening trial. BMJ 2014;348:g366

18 Gøtzsche PC, Hartling OJ, Nielsen M, Brodersen J, Jørgensen KJ. Breast screening: the facts-or maybe not. BMJ 2009;338:b86

19 Bagcchi S. India launches plan for national cancer screening programme. BMJ 2016;355:i5574

20 Sharma DC. Cancer data in India show new patterns. Lancet Oncol 2016;17(7):e272
21 Vanamail P. Epidemiological profile of female breast cancer in reproductive age-group and its association with maternal characteristics: A population-based observational study in India. Cancer 2019;4:28

22 Sandhu GS, Erqou S, Patterson H, Mathew A. Prevalence of triple-negative breast cancer in india: systematic review and meta-analysis. J Glob Oncol 2016;2(6):412-421

23 Holm J, Humphreys K, Li J, et al. Risk factors and tumor characteristics of interval cancers by mammographic density. J Clin Oncol 2015;33(9):1030-1037

24 Wilson J, Junger G. Principles and practice of screening for disease. Geneva: World Health Organization; 1968 (https:// apps.who.int/iris/bitstream/handle/10665/37650/WHO_PHP_ 34.pdf?sequence=17).

25 Sankaranarayanan R, Ramadas K, Thara S, et al. Clinical breast examination: preliminary results from a cluster randomized controlled trial in India. J Natl Cancer Inst 2011;103(19):1476-1480 